\title{
Probability distribution solutions of a general linear equation of infinite order, II
}

\author{
by Tomasz Kochanek and Janusz Morawiec (Katowice)
}

\begin{abstract}
Let $(\Omega, \mathcal{A}, P)$ be a probability space and let $\tau: \mathbb{R} \times \Omega \rightarrow \mathbb{R}$ be a mapping strictly increasing and continuous with respect to the first variable, and $\mathcal{A}$-measurable with respect to the second variable. We discuss the problem of existence of probability distribution solutions of the general linear equation

$$
F(x)=\int_{\Omega} F(\tau(x, \omega)) P(d \omega) .
$$

We extend our uniqueness-type theorems obtained in Ann. Polon. Math. 95 (2009), 103-114.
\end{abstract}

1. Introduction. Throughout the paper, $(\Omega, \mathcal{A}, P)$ is a probability space and $\tau: \mathbb{R} \times \Omega \rightarrow \mathbb{R}$ is a mapping such that for every $x \in \mathbb{R}$ the function $\tau(x, \cdot)$ is $\mathcal{A}$-measurable, and for every $\omega \in \Omega$ the function $\tau(\cdot, \omega)$ is strictly increasing and continuous.

We investigate the set of probability distribution (p.d.) solutions of the linear functional equation

$$
F(x)=\int_{\Omega} F(\tau(x, \omega)) P(d \omega)
$$

extending the results obtained in $[\mathrm{KM}]$; for the background of equation 1.1 see the references therein.

As explained in detail in [KM, $\S 2]$, we may restrict our considerations to the case where

$$
\{x \in \mathbb{R}: \tau(x, \omega)=x \text { for almost all } \omega \in \Omega\}=\emptyset .
$$

This follows from [MR, Theorem 2]; also by that theorem, we know that (1.2) forces every p.d. solution $F$ of 1.1 to be automatically continuous.

2010 Mathematics Subject Classification: Primary 60E05, 39B12; Secondary 39B22.

Key words and phrases: linear functional equations, iterative functional equations, probability distribution solutions. 
From now on we assume 1.2 . For any interval $J \subset \mathbb{R}$ we define $\mathcal{C}(J)=\{F: \mathbb{R} \rightarrow[0,1] \mid F$ is a weakly increasing (and continuous) solution of (1) such that $F(\inf J)=0$ and $F(\sup J)=1$, with the notation $F(-\infty)=\lim _{x \rightarrow-\infty} F(x)$ and $F(+\infty)=\lim _{x \rightarrow+\infty} F(x)$.

We say that a set $S \subset \mathbb{R}$ is $\tau$-invariant if $S \neq \emptyset$ and for every $x \in S$ we have $\tau(x, \omega) \in S$ for almost all $\omega \in \Omega$. Put

$$
\begin{aligned}
& \mathcal{S}_{1}=\{I \subset \mathbb{R}: I \text { is a minimal compact } \tau \text {-invariant interval }\}, \\
& \mathcal{S}_{2}=\left\{I \subset \mathbb{R} \backslash \bigcup \mathcal{S}_{1}: I \text { is a maximal } \tau \text {-invariant half-line }\right\},
\end{aligned}
$$

and $\mathcal{S}=\mathcal{S}_{1} \cup \mathcal{S}_{2}$. In view of the definition and our assumption (1.2), the family $\mathcal{S}$ consists of pairwise disjoint non-degenerate closed proper subintervals of $\mathbb{R}$. Therefore, since $\bigcup \mathcal{S}_{1}$ is closed (cf. the proof of [KM, Claim 7]), $\cup \mathcal{S}$ is closed as well. This implies that $\mathbb{R} \backslash \bigcup \mathcal{S}$ is a non-empty open set. Indeed, $\bigcup \mathcal{S}=\mathbb{R}$ would imply that the set of all end-points of the intervals from $\mathcal{S}$ is a countable perfect set, which is impossible.

By virtue of [KM, Corollary 2, Remarks 1 and 2], we find that:

(i) Every p.d. solution $F$ of $(1.1)$ is constant on each member of $\mathcal{S}$.

(ii) For each open component $J$ of the set $\mathbb{R} \backslash \bigcup \mathcal{S}$ the class $\mathcal{C}(J)$ has at most one element.

(iii) If $F$ is a p.d. solution of $(1.1)$ and $J$ is an open component of $\mathbb{R} \backslash \bigcup \mathcal{S}$ with $\mathcal{C}(J)=\{G\}$, then

$$
G=\frac{F-F(\inf J)}{F(\sup J)-F(\inf J)} .
$$

(iv) The existence of any p.d. solution of 1.1$)$ is equivalent to $\mathcal{C}(J) \neq \emptyset$ for at least one open component $J$ of $\mathbb{R} \backslash \cup \mathcal{S}$.

These four statements show that in order to describe every p.d. solution $F$ of equation (1.1) we should be able to decide whether $\mathcal{C}(J) \neq \emptyset$ and, if so, to describe the unique member of $\mathcal{C}(J)$, for every open component $J$ of $\mathbb{R} \backslash \bigcup \mathcal{S}$. This is the aim of the present paper.

2. Some lemmas. We start with two auxiliary lemmas which yield certain connections between solutions of any of the two inequalities:

$$
\begin{aligned}
& F_{0}(x) \geq \int_{\Omega} F_{0}(\tau(x, \omega)) P(d \omega), \\
& F_{0}(x) \leq \int_{\Omega} F_{0}(\tau(x, \omega)) P(d \omega)
\end{aligned}
$$

and solutions of equation (1.1). 
Lemma 2.1. If $F_{0}: \mathbb{R} \rightarrow[0,1]$ is an increasing solution of (2.1) (or $(2.2)$ ), then the sequence $\left(F_{n}\right)_{n \in \mathbb{N}}$ of functions $F_{n}: \mathbb{R} \rightarrow[0,1]$ defined by the formula

$$
F_{n}(x)=\int_{\Omega} F_{n-1}(\tau(x, \omega)) P(d \omega) \quad \text { for } n \in \mathbb{N}, x \in \mathbb{R}
$$

is decreasing (respectively increasing), hence it is pointwise convergent to a certain $F: \mathbb{R} \rightarrow[0,1]$.

Moreover, the function $F$ is either constant or

$$
\frac{F-F(-\infty)}{F(+\infty)-F(-\infty)} \in \mathcal{C}(\mathbb{R})
$$

Proof. If $F_{0}$ satisfies (2.1), then by the definition, $F_{1} \leq F_{0}$. In particular, $F_{1}(\tau(x, \omega)) \leq F_{0}(\tau(x, \omega))$ for all $x \in \mathbb{R}$ and $\omega \in \Omega$. After integration we get $F_{2} \leq F_{1}$ and, by induction, $F_{n} \leq F_{n-1}$ for every $n \in \mathbb{N}$. Analogously, if $F_{0}$ satisfies 2.2), then $F_{n-1} \leq F_{n}$ for every $n \in \mathbb{N}$. Let $F=\lim _{n \rightarrow \infty} F_{n}$. Since $F_{0}$ is increasing, each $F_{n}$ and $F$ itself are increasing as well. Moreover, $F$ satisfies (1.1). Thus, in view of [MR, Theorem 2], either $F$ is constant or (2.4) holds.

In the following we consider the product space $\left(\Omega^{\infty}, \mathcal{A}^{\infty}, P^{\infty}\right)$ and the iterates $\tau^{n}: \mathbb{R} \times \Omega^{\infty} \rightarrow \mathbb{R}$ defined (cf. [BJ], [BK], [D]) by putting

$$
\begin{aligned}
\tau^{1}\left(x, \omega_{1}, \ldots\right) & =\tau\left(x, \omega_{1}\right), \\
\tau^{n+1}\left(x, \omega_{1}, \ldots\right) & =\tau\left(\tau^{n}\left(x, \omega_{1}, \ldots\right), \omega_{n+1}\right) \quad \text { for } n \in \mathbb{N} .
\end{aligned}
$$

It is easily seen that for each $n \in \mathbb{N}$ we have

$$
\tau^{n+1}\left(x, \omega_{1}, \ldots\right)=\tau^{n}\left(\tau\left(x, \omega_{1}\right), \omega_{2}, \ldots\right)
$$

and the $n$th iterate $\tau^{n}(x, \omega)$ depends only on the first $n$ coordinates of $\omega$. Hence it is justified to write $\tau^{n}\left(x, \omega_{1}, \ldots, \omega_{m}\right)$ instead of $\tau^{n}\left(x, \omega_{1}, \ldots\right)$, if $m \geq n$.

Lemma 2.2. Assume $x_{0}, y_{0} \in \mathbb{R} \cup\{-\infty,+\infty\}$.

(i) If $x_{0} \in \mathbb{R}$ and $\left(F_{n}\right)_{n \in \mathbb{N}}$ is given by 2.3] with $F_{0}=\chi_{\left[x_{0},+\infty\right)}$, then

$$
F_{n}(x)=P^{\infty}\left(\tau^{n}(x, \omega) \geq x_{0}\right) \quad \text { for } n \in \mathbb{N}, x \in \mathbb{R} .
$$

If $\left(x_{0}, y_{0}\right)$ is a component of $\mathbb{R} \backslash \bigcup \mathcal{S}$, then $F_{0}$ satisfies (2.1).

(ii) If $y_{0} \in \mathbb{R}$ and $\left(F_{n}\right)_{n \in \mathbb{N}}$ is given by 2.3] with $F_{0}=\chi_{\left[y_{0},+\infty\right)}$, then

$$
F_{n}(x)=P^{\infty}\left(\tau^{n}(x, \omega) \geq y_{0}\right) \quad \text { for } n \in \mathbb{N}, x \in \mathbb{R} .
$$

If $\left(x_{0}, y_{0}\right)$ is a component of $\mathbb{R} \backslash \bigcup \mathcal{S}$, then $F_{0}$ satisfies 2.2 .

Proof. Since the proofs of both assertions (i) and (ii) are similar, we show only the first one. 
For any $x \in \mathbb{R}$ we have

$$
\begin{aligned}
F_{1}(x) & =\int_{\Omega} \chi_{\left[x_{0},+\infty\right)}\left(\tau\left(x, \omega_{1}\right)\right) P\left(d \omega_{1}\right) \\
& =P\left(\tau\left(x, \omega_{1}\right) \geq x_{0}\right)=P^{\infty}\left(\tau^{1}\left(x, \omega_{1}, \ldots\right) \geq x_{0}\right) .
\end{aligned}
$$

Assuming that the desired formula holds true for a fixed $n \in \mathbb{N}$ and every $x \in \mathbb{R}$, we get

$$
\begin{aligned}
F_{n+1}(x) & =\int_{\Omega} F_{n}\left(\tau\left(x, \omega_{1}\right)\right) P\left(d \omega_{1}\right) \\
& =\int_{\Omega} P^{n}\left(\tau^{n}\left(\tau\left(x, \omega_{1}\right), \omega_{2}, \ldots, \omega_{n+1}\right) \geq x_{0}\right) P\left(d \omega_{1}\right) \\
& =\int_{\Omega} P^{n}\left(\tau^{n+1}\left(x, \omega_{1}, \ldots, \omega_{n+1}\right) \geq x_{0}\right) P\left(d \omega_{1}\right) \\
& =P^{n+1}\left(\tau^{n+1}\left(x, \omega_{1}, \ldots, \omega_{n+1}\right) \geq x_{0}\right) \\
& =P^{\infty}\left(\tau^{n+1}\left(x, \omega_{1}, \ldots\right) \geq x_{0}\right) .
\end{aligned}
$$

Now, suppose $\left(x_{0}, y_{0}\right)$ is a component of $\mathbb{R} \backslash \bigcup \mathcal{S}$. Then $x_{0}$ is a right end-point of some $\tau$-invariant interval. Hence $\tau\left(x_{0}, \omega\right) \leq x_{0}$ for almost all $\omega \in \Omega$. Therefore, if $x<x_{0}$ then $\tau(x, \omega)<\tau\left(x_{0}, \omega\right) \leq x_{0}$ for almost all $\omega \in \Omega$, hence $F_{0}(\tau(x, \omega))=0$ for almost all $\omega \in \Omega$ and (2.1) holds; if $x \geq x_{0}$ then $F_{0}(x)=1$ and again (2.1) holds.

3. The case $\mathcal{S} \neq \emptyset$. Obviously, recursion $(2.3)$ may produce a nontrivial solution of equation (1.1) only if the initial function $F_{0}$ is nonconstant. Lemma 2.2 guarantees that in the case $\mathcal{S} \neq \emptyset$ it is always possible to find a suitable solution of $(2.1)$ or $(2.2)$. We will exploit this fact in the next lemma.

Throughout this section we assume $\mathcal{S} \neq \emptyset$.

Lemma 3.1. Assume $J=\left(x_{0}, y_{0}\right)$ is a component of $\mathbb{R} \backslash \bigcup \mathcal{S}$.

(i) If $x_{0} \in \mathbb{R}, y_{0} \in \mathbb{R},\left(F_{n}\right)_{n \in \mathbb{N}}$ is given by $(2.3)$ with $F_{0}=\chi_{\left[x_{0},+\infty\right)}$ and $F=\lim _{n \rightarrow \infty} F_{n}$, then $\mathcal{C}(J)=\{F\}$.

(ii) If $x_{0} \in \mathbb{R}, y_{0}=+\infty,\left(F_{n}\right)_{n \in \mathbb{N}}$ is given by 2.3 with $F_{0}=\chi_{\left[x_{0},+\infty\right)}$ and $F=\lim _{n \rightarrow \infty} F_{n}$, then $F \neq 0$ implies $\mathcal{C}(J)=\{F\}$, whereas $F=0$ implies $\mathcal{C}(J)=\emptyset$.

(iii) If $x_{0}=-\infty, y_{0} \in \mathbb{R},\left(F_{n}\right)_{n \in \mathbb{N}}$ is given by (2.3) with $F_{0}=\chi_{\left[y_{0},+\infty\right)}$ and $F=\lim _{n \rightarrow \infty} F_{n}$, then $F \neq 1$ implies $\mathcal{C}(J)=\{F\}$, whereas $F=1$ implies $\mathcal{C}(J)=\emptyset$.

Proof. (i) Since $J$ is a bounded component of $\mathbb{R} \backslash \bigcup \mathcal{S}$, we infer that $x_{0}$ and $y_{0}$ are (respectively right and left) end-points of some $\tau$-invariant 
intervals. Consequently,

$$
P\left(\tau(x, \omega) \leq x_{0}\right)=1, \quad P\left(\tau(y, \omega) \geq y_{0}\right)=1 \quad \text { for } x \leq x_{0}, y \geq y_{0} .
$$

A simple induction yields

$P^{\infty}\left(\tau^{n}(x, \omega) \leq x_{0}\right)=1, P^{\infty}\left(\tau^{n}(y, \omega) \geq y_{0}\right)=1 \quad$ for $n \in \mathbb{N}, x \leq x_{0}, y \geq y_{0}$, which, in the light of Lemma 2.2, means nothing else than

$$
\left.F_{n}\right|_{\left(-\infty, x_{0}\right]}=0 \quad \text { and }\left.\quad F_{n}\right|_{\left[y_{0},+\infty\right)}=1 \quad \text { for } n \in \mathbb{N} .
$$

By Lemma 2.1, $F \in \mathcal{C}(J)$.

(ii) Analogously to the proof of (i) we can show that $\left.F\right|_{\left(-\infty, x_{0}\right]}=0$ and either $F$ is constant or the function $F / F(+\infty)$ belongs to $\mathcal{C}(J)$. Now it is enough to prove that $\mathcal{C}(J) \neq \emptyset$ implies $F(+\infty)=1$.

Let $G \in \mathcal{C}(J)$. Obviously, $G \leq F_{0}$. Therefore

$$
G(x)=\int_{\Omega} G(\tau(x, \omega)) P(d \omega) \leq \int_{\Omega} F(\tau(x, \omega)) P(d \omega)=F_{1}(x)
$$

for $x \in \mathbb{R}$ and further, by induction, $G \leq F_{n}$ for every $n \in \mathbb{N}$. Hence $G \leq F$, which implies $F(+\infty)=1$.

(iii) The proof runs analogously to the proof of (ii).

As a consequence of Lemmas 2.2 and 3.1 we obtain the following theorem.

Theorem 3.2. Assume $\mathcal{S} \neq \emptyset$ and $J=\left(x_{0}, y_{0}\right)$ is a component of $\mathbb{R} \backslash \bigcup \mathcal{S}$.

(i) If $x_{0} \in \mathbb{R}$ and $y_{0} \in \mathbb{R}$, then $\mathcal{C}(J) \neq \emptyset$. The unique member of $\mathcal{C}(J)$ is given by $F=\lim _{n \rightarrow \infty} F_{n}$, where $\left(F_{n}\right)_{n \in \mathbb{N}}$ is defined by 2.3) with $F_{0}=\chi_{\left[x_{0},+\infty\right)}$.

(ii) If $x_{0} \in \mathbb{R}$ and $y_{0}=+\infty$, then $\mathcal{C}(J) \neq \emptyset$ if and only if

$$
\lim _{x \rightarrow+\infty} \lim _{n \rightarrow \infty} P^{\infty}\left(\tau^{n}(x, \omega) \geq x_{0}\right)>0 .
$$

In that case the unique member of $\mathcal{C}(J)$ is given by $F=\lim _{n \rightarrow \infty} F_{n}$, where $\left(F_{n}\right)_{n \in \mathbb{N}}$ is defined by (2.3) with $F_{0}=\chi_{\left[x_{0},+\infty\right)}$.

(iii) If $x_{0}=-\infty, y_{0} \in \mathbb{R}$, then $\mathcal{C}(J) \neq \emptyset$ if and only if

$$
\lim _{y \rightarrow-\infty} \lim _{n \rightarrow \infty} P^{\infty}\left(\tau^{n}(y, \omega)<y_{0}\right)>0 .
$$

In that case the unique member of $\mathcal{C}(J)$ is given by $F=\lim _{n \rightarrow \infty} F_{n}$, where $\left(F_{n}\right)_{n \in \mathbb{N}}$ is defined by 2.3. with $F_{0}=\chi_{\left[y_{0},+\infty\right)}$.

Unfortunately, the two necessary and sufficient conditions appearing in assertions (ii) and (iii) are hard to verify in concrete situations. Nevertheless, there are clear conditions which are necessary for $\mathcal{C}(J) \neq \emptyset$. 
Theorem 3.3. Assume $J=\left(x_{0}, y_{0}\right)$ is a component of $\mathbb{R} \backslash \bigcup \mathcal{S}$.

(i) If $x_{0} \in \mathbb{R}, y_{0}=+\infty$ and $\mathcal{C}(J) \neq \emptyset$, then almost all functions $\tau(\cdot, \omega)$ are unbounded from above.

(ii) If $x_{0}=-\infty, y_{0} \in \mathbb{R}$ and $\mathcal{C}(J) \neq \emptyset$, then almost all functions $\tau(\cdot, \omega)$ are unbounded from below.

Proof. Since the proofs of (i) and (ii) are similar, we only show (i).

Suppose that there exists $M \in \mathbb{R}$ such that $\alpha=P(\tau(\cdot, \omega) \leq M)>0$. Define a sequence $\left(\xi_{n}\right)_{n \geq 0}$ by the formula

$$
\xi_{n}=\inf \left\{x \in \mathbb{R}: F_{n}(x)=1\right\},
$$

where $\left(F_{n}\right)_{n \in \mathbb{N}}$ is given by $(2.3)$ with $F_{0}=\chi_{\left[x_{0},+\infty\right)}$ (we put $\xi_{n}=+\infty$ if the underlying set is empty). By Lemma 2.2(i), $F_{0}$ satisfies (2.1), hence Lemma 2.1 implies that the sequence $\left(F_{n}\right)_{n \in \mathbb{N}}$ is decreasing. Therefore $\left(\xi_{n}\right)_{n \geq 0}$ is increasing, and since $\xi_{0}=x_{0}$, it follows that $x_{0} \leq \xi_{n-1} \leq \xi_{n}$ for $n \in \mathbb{N}$.

We will show that

$$
\xi_{n}=\inf \left\{x \in \mathbb{R}: P\left(\tau(x, \omega) \geq \xi_{n-1}\right)=1\right\} \quad \text { for } n \in \mathbb{N} .
$$

The case $\xi_{n}=+\infty$ is trivial, since then formula 2.3 implies that there is no $x \in \mathbb{R}$ with $P\left(\tau(x, \omega) \geq \xi_{n-1}\right)=1$. Thus we may assume $\xi_{n}<+\infty$, and consequently $\xi_{n-1}<+\infty$. Denote the right-hand side of $(3.2)$ by $\eta_{n}$. The equality

$$
F_{n}(x)=\int_{\Omega} F_{n-1}(\tau(x, \omega)) P(d \omega)=1,
$$

jointly with (3.1), implies that $P\left(\tau(x, \omega) \geq \xi_{n-1}\right)=1$ for every $x>\xi_{n}$. Therefore $P\left(\tau\left(\xi_{n}, \omega\right) \geq \xi_{n-1}\right)=1$, i.e. $\xi_{n} \geq \eta_{n}$. For the converse inequality fix any $x \in \mathbb{R}$ with $P\left(\tau(x, \omega) \geq \xi_{n-1}\right)=1$. Then (3.3) yields $F_{n}(x)=1$, hence $\xi_{n} \leq x$. This shows that $\xi_{n} \leq \eta_{n}$.

Let $\xi=\lim _{n \rightarrow \infty} \xi_{n}$. If $\xi$ were finite then, in view of $(3.2)$, we would have $\tau(\xi, \omega) \geq \xi$ for almost all $\omega \in \Omega$, which means that $\xi$ is a left end-point of some $\tau$-invariant interval. This, however, is impossible, since $\xi \geq x_{0}$ and $\left(x_{0},+\infty\right) \subset \mathbb{R} \backslash \cup \mathcal{S}$. Therefore $\xi=+\infty$, hence there is $n \in \mathbb{N}$ such that $\xi_{n}>M$. In view of (3.1), we have $F_{n}(M)<1$. For every $x \in \mathbb{R}$ we thus obtain

$$
\begin{aligned}
F_{n+1}(x) & =\int_{\Omega} F_{n}(\tau(x, \omega)) P(d \omega)=\int_{\tau(x, \omega) \leq M}+\int_{\tau(x, \omega)>M} \\
& \leq \alpha F_{n}(M)+(1-\alpha)<1 .
\end{aligned}
$$

Consequently, $F_{n+1}(+\infty)<1$. Hence we also have

$$
\lim _{x \rightarrow+\infty} \lim _{n \rightarrow \infty} F_{n}(x)<1,
$$

which in the light of Lemma 2.2(i) and Theorem 3.2(ii) implies $\mathcal{C}(J)=\emptyset$. Thus the proof has been completed. 
4. The case $\mathcal{S}=\emptyset$. Throughout this section we assume $\mathcal{S}=\emptyset$.

Lemma 4.1. If $F \in \mathcal{C}(\mathbb{R})$, then $F(\mathbb{R}) \subset(0,1)$.

Proof. Put $x_{0}=\inf \{x \in \mathbb{R}: F(x)=1\}$ and suppose $x_{0} \in \mathbb{R}$. Then it follows from

$$
1=F\left(x_{0}\right)=\int_{\Omega} F\left(\tau\left(x_{0}, \omega\right)\right) P(d \omega)
$$

that $F\left(\tau\left(x_{0}, \omega\right)\right)=1$ for almost all $\omega \in \Omega$, thus $\tau\left(x_{0}, \omega\right) \geq x_{0}$ for almost all $\omega \in \Omega$, which contradicts $\mathcal{S}=\emptyset$. Similarly we can prove that $F(x)>0$ for every $x \in \mathbb{R}$.

Lemma 4.2. If $x_{0} \in \mathbb{R}, F \in \mathcal{C}(\mathbb{R})$ and $\left(F_{n}\right)_{n \in \mathbb{N}}$ is given by (2.3) with $F_{0}=\chi_{\left[x_{0},+\infty\right)}$, then

$$
\frac{F(x)-F\left(x_{0}\right)}{1-F\left(x_{0}\right)} \leq F_{n}(x) \leq \frac{F(x)}{F\left(x_{0}\right)} \quad \text { for } n \in \mathbb{N}, x \in \mathbb{R} .
$$

Proof. Iterating equation (1.1) and using Lemma 2.2(i) we get

$$
\begin{aligned}
F(x) & =\int_{\Omega^{\infty}} F\left(\tau^{n}(x, \omega)\right) P^{\infty}(d \omega)=\int_{\tau^{n}\left(x_{0}, \omega\right) \geq x_{0}}+\int_{\tau^{n}\left(x_{0}, \omega\right)<x_{0}} \\
& \leq P^{\infty}\left(\tau^{n}(x, \omega) \geq x_{0}\right)+F\left(x_{0}\right) P^{\infty}\left(\tau^{n}(x, \omega)<x_{0}\right) \\
& =F_{n}(x)+F\left(x_{0}\right)\left(1-F_{n}(x)\right)
\end{aligned}
$$

for $n \in \mathbb{N}, x \in \mathbb{R}$. By Lemma 4.1 we obtain the first estimate in 4.1). To show the second one we write

$$
\begin{aligned}
F(x) & \geq \int_{\tau^{n}\left(x_{0}, \omega\right) \geq x_{0}} F\left(\tau^{n}(x, \omega)\right) P^{\infty}(d \omega) \\
& \geq F\left(x_{0}\right) P^{\infty}\left(\tau^{n}(x, \omega) \geq x_{0}\right)=F\left(x_{0}\right) F_{n}(x)
\end{aligned}
$$

for $n \in \mathbb{N}, x \in \mathbb{R}$.

LEMma 4.3. If $x_{0} \in \mathbb{R}$ and $\left(F_{n}\right)_{n \in \mathbb{N}}$ is given by 2.3 with $F_{0}=\chi_{\left[x_{0},+\infty\right)}$, then the function $\underline{F}: \mathbb{R} \rightarrow[0,1]$ defined by

$$
\underline{F}(x)=\liminf _{n \rightarrow \infty} F_{n}(x)
$$

is increasing and satisfies

$$
\underline{F}(x) \geq \int_{\Omega} \underline{F}(\tau(x, \omega)) P(d \omega),
$$

whereas the function $\bar{F}: \mathbb{R} \rightarrow[0,1]$ defined by

$$
\bar{F}(x)=\limsup _{n \rightarrow \infty} F_{n}(x)
$$

is increasing and satisfies

$$
\bar{F}(x) \leq \int_{\Omega} \bar{F}(\tau(x, \omega)) P(d \omega) .
$$


Proof. It is obvious that $\underline{F}$ and $\bar{F}$ are increasing. Inequalities 4.2 and 4.3 immediately follow from the Fatou lemma applied to the sequences $\left(F_{n}\right)_{n \in \mathbb{N}}$ and $\left(1-F_{n}\right)_{n \in \mathbb{N}}$, respectively.

From now on $x_{0} \in \mathbb{R}$ is fixed and $\underline{F}, \bar{F}$ stand for the two functions defined in Lemma 4.3 .

LEMMA 4.4. In each of the following cases:

(a) $\left(F_{n}\right)_{n \in \mathbb{N}}$ is given by 2.3 with $F_{0}=\underline{F}$ and $F=\lim _{n \rightarrow \infty} F_{n}$;

(b) $\left(F_{n}\right)_{n \in \mathbb{N}}$ is given by 2.3. with $F_{0}=\bar{F}$ and $F=\lim _{n \rightarrow \infty} F_{n}$, we have:

(i) If $F$ is non-constant, then $\mathcal{C}(\mathbb{R})=\{F\}$.

(ii) If $F$ is constant, then $\mathcal{C}(\mathbb{R})=\emptyset$.

Proof. Both in case (a) and (b), Lemma 4.3 , jointly with Lemma 2.1 , implies that $F: \mathbb{R} \rightarrow[0,1]$ is a well-defined function such that (2.4) holds provided $F$ is non-constant. Now it is enough to show that $\mathcal{C}(\mathbb{R}) \neq \emptyset$ implies both $F(-\infty)=0$ and $F(+\infty)=1$.

Let $G \in \mathcal{C}(\mathbb{R})$. By Lemma 4.2 ,

$$
\frac{G(x)-G\left(x_{0}\right)}{1-G\left(x_{0}\right)} \leq \underline{F}(x) \leq \bar{F}(x) \leq \frac{G(x)}{G\left(x_{0}\right)} \quad \text { for } x \in \mathbb{R} .
$$

Substituting $\tau(x, \omega)$ for $x$, integrating both sides and applying a simple induction we arrive at the inequalities

$$
\frac{G(x)-G\left(x_{0}\right)}{1-G\left(x_{0}\right)} \leq F_{n}(x) \leq \frac{G(x)}{G\left(x_{0}\right)} \quad \text { for } x \in \mathbb{R},
$$

where $F_{n}$ may be defined either as in case (a) or as in case (b). In both cases we may pass to the limits as $n \rightarrow \infty$ and then $x \rightarrow \pm \infty$ to obtain $F(-\infty)=0$ and $F(+\infty)=1$.

As a consequence of Lemma 4.4 we obtain the following theorem.

TheOREM 4.5. We have $\mathcal{C}(\mathbb{R}) \neq \emptyset$ if and only if the limit

$$
F(x)=\lim _{n \rightarrow \infty} P^{\infty}\left(\tau^{n}(x, \omega)>x_{0}\right)
$$

exists for every $x \in \mathbb{R}$ and the function $F$ is a probability distribution. In that case $\mathcal{C}(J)=\{F\}$.

Proof. Assume first that formula (4.4) defines a p.d. function $F$ : $\mathbb{R} \rightarrow[0,1]$. Then, by virtue of Lemma $2.2(\mathrm{i})$, we infer that $F=\lim _{n \rightarrow \infty} F_{n}$, where $\left(F_{n}\right)_{n \in \mathbb{N}}$ is given by $(2.3)$ with $F_{0}=\chi_{\left[x_{0},+\infty\right)}$. Thus it follows immediately from $(2.3)$ that $F$ is a solution of $(1.1)$. Hence $\mathcal{C}(\mathbb{R})=\{F\}$.

Now, assume that there exists a function $G \in \mathcal{C}(\mathbb{R})$ and let us distinguish cases (a) and (b) of Lemma 4.4. By using Lemmas 4.3 and 2.2(i), we obtain what follows. 
CASE (a). For any $x \in \mathbb{R}$ we have

$$
F_{1}(x)=\int_{\Omega} \underline{F}(\tau(x, \omega)) P(d \omega) \leq \underline{F}(x)=\liminf _{n \rightarrow \infty} P^{\infty}\left(\tau^{n}(x, \omega) \geq x_{0}\right) .
$$

Since Lemmas 2.1 and 4.3 imply that the sequence $\left(F_{n}\right)_{n \in \mathbb{N}}$ is decreasing, we infer that

$$
F_{m}(x) \leq \liminf _{n \rightarrow \infty} P^{\infty}\left(\tau^{n}(x, \omega) \geq x_{0}\right) \quad \text { for } m \in \mathbb{N}, x \in \mathbb{R} .
$$

By Lemma 4.4. $\lim _{m \rightarrow \infty} F_{m}=G$ and hence 4.5 yields

$$
G(x) \leq \liminf _{n \rightarrow \infty} P^{\infty}\left(\tau^{n}(x, \omega) \geq x_{0}\right) \quad \text { for } x \in \mathbb{R} .
$$

CAse (b). For any $x \in \mathbb{R}$ we have

$$
F_{1}(x)=\int_{\Omega} \bar{F}(\tau(x, \omega)) P(d \omega) \geq \bar{F}(x)=\limsup _{n \rightarrow \infty} P^{\infty}\left(\tau^{n}(x, \omega) \geq x_{0}\right) .
$$

Since Lemmas 2.1 and 4.3 imply that the sequence $\left(F_{n}\right)_{n \in \mathbb{N}}$ is increasing, we infer that

$$
F_{m}(x) \geq \limsup _{n \rightarrow \infty} P^{\infty}\left(\tau^{n}(x, \omega) \geq x_{0}\right) \quad \text { for } m \in \mathbb{N}, x \in \mathbb{R} .
$$

By Lemma 4.4. $\lim _{m \rightarrow \infty} F_{m}=G$ and hence 4.7) yields

$$
G(x) \geq \limsup _{n \rightarrow \infty} P^{\infty}\left(\tau^{n}(x, \omega) \geq x_{0}\right) \quad \text { for } x \in \mathbb{R} .
$$

Inequalities (4.6) and 4.8) show that the limit $F(x)$ given by (4.4) exists and for every $x \in \mathbb{R}$ we have $F(x)=G(x)$, which completes the proof.

The last result, which is analogous to Theorem 3.3 , gives a necessary condition for $\mathcal{C}(\mathbb{R}) \neq \emptyset$.

THEOREM 4.6. If $\mathcal{C}(\mathbb{R}) \neq \emptyset$, then almost all functions $\tau(\cdot, \omega)$ are unbounded from below and from above.

Proof. Suppose first that there exists $M \in \mathbb{R}$ such that $P(\tau(\cdot, \omega) \leq M)$ $>0$. Let $\left(F_{n}\right)_{n \in \mathbb{N}}$ and $F$ be as in Lemma 4.4(a). Define a sequence $\left(\xi_{n}\right)_{n \geq 0}$ by formula (3.1). If $\xi_{0}=-\infty$ then obviously we have $\underline{F}=F=1$, hence Lemma 4.4(ii) implies $\mathcal{C}(\mathbb{R})=\emptyset$. Thus we may assume $\xi_{0}>-\infty$. In view of inequality (4.2) and Lemma 2.1, the sequence $\left(F_{n}\right)_{n \in \mathbb{N}}$ is decreasing. As in the proof of Theorem 3.3 , we deduce that

$$
\xi_{n}=\inf \left\{x \in \mathbb{R}: P\left(\tau(x, \omega) \geq \xi_{n-1}\right)=1\right\} \geq \xi_{n-1} \quad \text { for } n \in \mathbb{N},
$$

and we may consider $\xi=\lim _{n \rightarrow \infty} \xi_{n}$. If $\xi$ were finite then we would have $\tau(\xi, \omega) \geq \xi$ for almost all $\omega \in \Omega$, which contradicts the fact that $\mathcal{S}=\emptyset$. Therefore $\xi=+\infty$ and, by the argument of the proof of Theorem 3.3 , we infer that $F(+\infty)<1$. Hence, in view of Lemma 4.4, we must have $\mathcal{C}(\mathbb{R})=\emptyset$.

Now, suppose that for some $m \in \mathbb{R}$ we have $\alpha=P(\tau(\cdot, \omega) \geq m)>0$. Let $\left(F_{n}\right)_{n \in \mathbb{N}}$ and $F$ be as in Lemma $4.4\left(\right.$ b). Define a sequence $\left(\nu_{n}\right)_{n \geq 0}$ by 
the formula

$$
\nu_{n}=\sup \left\{x \in \mathbb{R}: F_{n}(x)=0\right\}
$$

$\left(\nu_{n}=-\infty\right.$ if the underlying set is empty). If $\nu_{0}=+\infty$ then obviously $\bar{F}=F=0$, hence Lemma 4.4 (ii) implies $\mathcal{C}(\mathbb{R})=\emptyset$. Thus we may assume $\nu_{0}<+\infty$.

In view of inequality (4.3) and Lemma 2.1, the sequence $\left(F_{n}\right)_{n \in \mathbb{N}}$ is increasing. Therefore $\left(\nu_{n}\right)_{n \geq 0}$ is decreasing: $\nu_{n} \leq \nu_{n-1} \leq \nu_{0}<+\infty$ for $n \in \mathbb{N}$. Just as above we conclude that $\lim _{n \rightarrow \infty} \nu_{n}=-\infty$, hence $\nu_{n}<m$ for some $n \in \mathbb{N}$. By $(4.9)$, we have $F_{n}(m)>0$. For every $x \in \mathbb{R}$ we thus obtain

$$
F_{n+1}(x)=\int_{\Omega} F_{n}(\tau(x, \omega)) P(d \omega) \geq \int_{\tau(x, \omega) \geq m} \geq \alpha F_{n}(m)>0 .
$$

Consequently, $F_{n}(-\infty)>0$ and also $F(-\infty)>0$. In view of Lemma 4.4 . we infer that $\mathcal{C}(\mathbb{R})=\emptyset$.

Acknowledgments. This research has been supported by the scholarship from the UPGOW project co-financed by the European Social Fund (the first author), and the Silesian University Mathematics Department Functional Equations program (the second author).

\section{References}

[BJ] K. Baron and W. Jarczyk, Random-valued functions and iterative functional equations, Aequationes Math. 67 (2004), 140-153.

[BK] K. Baron and M. Kuczma, Iteration of random-valued functions on the unit interval, Colloq. Math. 37 (1977), 263-269.

[D] Ph. Diamond, A stochastic functional equation, Aequationes Math. 15 (1977), $225-233$.

[KM] T. Kochanek and J. Morawiec, Probability distribution solutions of a general linear equation of infinite order, Ann. Polon. Math. 95 (2009), 103-114.

[MR] J. Morawiec and L. Reich, The set of probability distribution solutions of a linear functional equation, ibid. 93 (2008), 253-261.

Tomasz Kochanek, Janusz Morawiec

Institute of Mathematics

Silesian University

40-007 Katowice, Poland

E-mail: tkochanek@math.us.edu.pl

morawiec@math.us.edu.pl

Received 20.7.2009

and in final form 29.1.2010 